\title{
Agonists for G-protein-coupled receptor 84 (GPR84) alter cellular morphology and motility but do not induce pro- inflammatory responses in microglia
}

\author{
Li Wei ${ }^{1,2,3}$, Kyohei Tokizane ${ }^{1}$, Hiroyuki Konishi ${ }^{1}$, Hua-Rong Yu ${ }^{3}$ and Hiroshi Kiyama ${ }^{1 *}$ [D
}

\begin{abstract}
Background: Several G-protein-coupled receptors (GPCRs) have been shown to be important signaling mediators between neurons and glia. In our previous screening for identification of nerve injury-associated GPCRs, G-proteincoupled receptor 84 (GPR84) mRNA showed the highest up-regulation by microglia after nerve injury. GPR84 is a pro-inflammatory receptor of macrophages in a neuropathic pain mouse model, yet its function in resident microglia in the central nervous system is poorly understood.

Methods: We used endogenous, natural, and surrogate agonists for GPR84 (capric acid, embelin, and 6-OAU, respectively) and examined their effect on mouse primary cultured microglia in vitro.

Results: 6-n-Octylaminouracil (6-OAU), embelin, and capric acid rapidly induced membrane ruffling and motility in cultured microglia obtained from C57BL/6 mice, although these agonists failed to promote microglial pro-inflammatory cytokine expression. Concomitantly, 6-OAU suppressed forskolin-induced increase of CAMP in cultured microglia. Pertussis toxin, an inhibitor of Gi-coupled signaling, completely suppressed 6-OAU-induced microglial membrane ruffling and motility. In contrast, no 6-OAU-induced microglial membrane ruffling and motility was observed in microglia from DBA/2 mice, a mouse strain that does not express functional GPR84 protein due to endogenous nonsense mutation of the GPR84 gene.
\end{abstract}

Conclusions: GPR84 mediated signaling causes microglial motility and membrane ruffling but does not promote pro-inflammatory responses. As GPR84 is a known receptor for medium-chain fatty acids, those released from damaged brain cells may be involved in the enhancement of microglial motility through GPR84 after neuronal injury.

Keywords: G-protein-coupled receptor 84, Pro-inflammatory response, Microglia morphology, Microglia motility, Fatty acid

\section{Background}

G-protein-coupled receptors (GPCRs) form the largest superfamily of membrane proteins, and several GPCRs are important signal mediators between neurons and glia, protecting neurons from pathological stressors that cause disease [1, 2]. To identify GPCRs implicated in neuronal survival and nerve regeneration after neuronal

\footnotetext{
* Correspondence: kiyama@med.nagoya-u.ac.jp

${ }^{1}$ Department of Functional Anatomy and Neuroscience, Nagoya University, Graduate School of Medicine, Nagoya, 65 Tsurumai-cho, Showa-ku, Nagoya, Aichi 466-8550, Japan

Full list of author information is available at the end of the article
}

injury, we previously performed a gene screen targeting GPCRs [3]. For this gene screen, we designed 274 primer sets for putative nonsensory GPCRs (including orphan receptors but excluding sensory GPCRs) and successfully identified several candidate GPCRs [3]. Among 274 genes, 29 genes were up-regulated by more than twofold after nerve injury. Further in situ hybridization secondary screening revealed that some of these receptors were specifically expressed and induced by microglia, and not neurons or astrocytes, in response to neuronal injury. This suggests that GPCRs and their ligands can be mediators for neuron-microglia interactions, particularly 
under traumatic or inflammatory conditions. Indeed, previous studies have demonstrated that $\mathrm{C}$ - $\mathrm{C}$ chemokine receptor type 5 (CCR5), CX3C chemokine receptor 1 (CX3CR1), and several purinergic receptors are expressed by microglia and play a variety of roles under physiological and pathological conditions [3-6].

In our previous GPCR screening study, mRNA encoding G-protein-coupled receptor 84 (GPR84) showed highest up-regulation (increased by approximately 47 -fold) in microglia after neuronal injury [3]. Other papers also reported GPR84 up-regulation in microglia in a mouse model of endotoxemia, experimental autoimmune encephalomyelitis, and Alzheimer's disease [7, 8]. GPR84 was first identified in myeloid cells by Wittenberger et al. [9], and separately by Yousefi et al. [10], and shown to be commonly expressed in species from lower vertebrates to humans. Interestingly a 2-bp frame-shift deletion in the second exon of the GPR84 gene has also been discovered in some classical inbred mouse strains. This deletion generates a premature stop codon, resulting in a truncated protein that lacks transmembrane domains 4-7 [11]. Fourteen mice strains including DBA, NOD, and SJL are homozygous for the deletion, but other strains including C57BL/6 are intact. A subsequent knockout strategy using C57BL/6 mice demonstrated no obvious abnormalities except for certain immune responses [12]. However, the involvement of GPR84 in experimental neuropathic pain was recently reported [13]. This study revealed that proinflammatory responses of macrophages recruited to injured nerves are suppressed in GPR84 KO mice after peripheral nerve injury, resulting in improvement of pain behavior. Subsequently, the authors concluded that GPR84 does not modulate microglial responses in the spinal dorsal horn, because GPR84 deficiency caused no alterations in microglial number and activation determined by ionized calcium-binding adapter molecule 1 and p-p38 immunostaining, respectively. Nonetheless, microglia responded to nerve injury with prominent upregulation of GPR84 mRNA in the neuropathic pain model [13], as well as our motor nerve injury model [3], in which circulating macrophages do not contribute to the microglial pool [14-16], and thereby conflicts with the conclusion that there is no functional consequence of GPR84 deficiency in microglia [13].

Previous studies have deorphanized and characterized GPR84 as a receptor for medium-chain fatty acids such as capric acids $[17,18]$. These papers also revealed that GPR84 activated chemotaxis and pro-inflammatory cytokine production in leukocyte and macrophage cell lines. Because some fatty acids regulate morphology, motility, and inflammatory responses of microglia [19-21], it is possible that a GPR84-mediated signal modulates microglial morphology and/or activity. Here, we addressed GPR84 function in primary cultured microglia using an endogenous ligand, capric acid (C10:0), a natural ligand, embelin (2,5-dihydroxy-3-undecyl-2,5-cyclohexadiene-1,4-dione), and a surrogate agonist, 6-noctylaminouracil (6-OAU).

\section{Methods \\ Animals}

C57BL/6 and DBA/2 mice were purchased from SLC Japan (Hamamatsu, Japan). Mice were housed with food and water available ad-libitum in a temperature $\left(23 \pm 1^{\circ}\right.$ C) - and humidity (50\%)-controlled environment on a 12/12-h light/dark cycle (lights on at $09.00 \mathrm{~h}$ ). All mice were maintained in accordance with the Guide for the Care and Use of Laboratory Animals (National Institutes of Health, 1996). The protocol for the animal experiment was approved by the Animal Ethics Committee of Nagoya University (Approval nos.: 26180, 27203, 28275).

\section{Materials}

6-OAU, embelin, and capric acid were provided by Ono Pharmaceutical Co. (Osaka, Japan). 6-OAU and capric acid were dissolved in absolute ethyl alcohol, and embelin in dimethyl sulfoxide. Solutions were aliquoted to avoid freeze-thawing and stored at $-80{ }^{\circ} \mathrm{C}$. A selective Gi-coupled signaling inhibitor, pertussis toxin (PTX), was purchased from Wako (\#168-22471; Osaka, Japan). Lipopolysaccharide (LPS) was purchased from SigmaAldrich (\#L4516; St Louis, MO, USA).

\section{Microglial culture}

Microglia were obtained from primary mixed cultures, in accordance with a previous report [22]. Briefly, meninges were removed from cerebra of C57BL/6 and DBA/ 2 mice at postnatal days $1-3$. Cerebral tissue was dispersed with $0.25 \%$ trypsin (Invitrogen, Carlsbad, CA, USA) and DNase I (Roche Applied Science, Indianapolis, IN, USA). Cells were filtered through $100 \mu \mathrm{m}$ nylon mesh, suspended in Dulbecco's Modified Eagle's medium (DMEM) supplemented with $10 \%$ fetal bovine serum (FBS) and $1 \%$ penicillin/streptomycin, and seeded into culture flasks. After 10-12 days in culture, detached microglia were collected and plated on 24- or 96-well culture dishes. Purity was > 98\% [23].

\section{Quantitative real-time RT-PCR (qPCR)}

Microglia were seeded into 96-well plates at a density of $3 \times 10^{4}$ cells/well in DMEM containing 10\% FBS and incubated overnight. After serum-starvation for $4 \mathrm{~h}$, microglia were stimulated with $100 \mathrm{ng} / \mathrm{mL}$ LPS in the presence or absence of various 6-OAU concentrations for 16 h. SYBR Green Cells-to-Ct Kit (Thermo Fisher Scientific, Waltham, MA, USA) was used to synthesize cDNA in accordance with the manufacturer's instructions. qPCR was performed using SYBR Green (Thermo Fisher 
Scientific) with the StepOnePlus Real-Time RT-PCR System (Thermo Fisher Scientific). The following primers were used: interleukin (IL)-1 $\beta$ forward: 5 '-ACAGAATATCAACCAACAAGTGATATTCTC-3'; IL-1 $\beta$ reverse: 5'GATTCTTTCCTTTGAGGCCCA-3'; IL-6 forward: 5' ATCCAGTTGCCTTCTTGGGACTGA-3'; IL-6 reverse: 5'-TAAGCCTCCGACTTGTGAAGTGGT-3'; IL-12 p35 forward: 5'-GACATCACACGGGACCAA-3'; IL-12 p35 reverse: 5'-AGTCCTCATAGATGCTACCAAG-3'; IL-12 p40 forward: 5'-TTCACGTGCTCATGGCT-3'; IL-12 p40 reverse: 5' -TTGGTCCAGTGTGACCTT-3'; chemokine (C-X-C motif) ligand 1 (CXCL1) forward: $5^{\prime}$ ACCCAAACCGAAGTCATAGC-3'; CXCL1 reverse: 5'TGGGGACACCTTTTAGCATC-3'; tumor necrosis factor (TNF)- $\alpha$ forward: 5 '-AGCCGATGGGTTGTACCTT GTCTA-3'; TNF- $\alpha$ reverse: 5'-TGAGATAGCAAATC GGCTGACGGT-3'; inducible nitric oxide synthase (iNOS) forward: 5'-GGCAGCCTGTGAGACCTTTG-3'; iNOS reverse: 5'-GAAGCGTTTCGGGATCTGAA-3'; glyceraldehyde-3-phosphate dehydrogenase (GAPDH) forward: 5'-CAAGGTCATCCCAGAGCTGA-3'; and GAPDH reverse: 5'-CGGCACGTCAGATCCACGAC-3' . qPCR reaction conditions were: 1 cycle of $95^{\circ} \mathrm{C}$ for $20 \mathrm{~s}$, followed by 40 cycles of $95^{\circ} \mathrm{C}$ for $3 \mathrm{~s}$ and $60^{\circ} \mathrm{C}$ for $30 \mathrm{~s}$. At the end of PCR, samples were subjected to melting analysis to confirm amplicon specificity. Relative gene expression ( $\Delta \mathrm{Ct}$ value) was calculated based on threshold cycle $(\mathrm{Ct})$ of the reference gene $(\mathrm{GAPDH})$ and target gene $\left(\Delta \mathrm{Ct}=\mathrm{Ct}_{\text {target }}\right.$ gene $-\mathrm{Ct}_{\text {reference gene) }} . \Delta \Delta \mathrm{Ct}$ was calculated as $\Delta \mathrm{Ct}_{\mathrm{LPS}}$ treatment $-\Delta \mathrm{Ct}_{\mathrm{LPS}}+6$-OAU treatment with $2^{-\Delta \Delta \mathrm{Ct}}$ determined as fold change [24].

\section{Staining cultured microglia}

Microglia were seeded onto glass coverslips in 24-well culture dishes and incubated overnight at $37^{\circ} \mathrm{C}$. Cultured microglia were serum-starved for $4 \mathrm{~h}$, and then stimulated with 6-OAU, embelin, and capric acid at the indicated concentration. For inhibition of Gi-coupled signaling, microglia were pretreated with PTX $(100 \mathrm{ng} / \mathrm{mL}, 4 \mathrm{~h})$. Microglia were fixed in phosphate-buffered saline (PBS), $\mathrm{pH} 7.4$, containing $4 \%$ paraformaldehyde for $10 \mathrm{~min}$ at room temperature. Immunostaining was performed according to our previous report [23], using rabbit antinuclear factor kappa B (NF-kB) p65 antibody (1:100; \#L1207; Santa Cruz Biotech, Santa Cruz, California, USA) and Alexa Fluor 488-labeled anti-rabbit IgG (1:1000; Invitrogen) as primary and secondary antibodies, respectively. Actin was visualized using Alexa Fluor 594-labeled phalloidin (1:100; \#A12381; Invitrogen). Nuclei were stained with 4',6-diamidino-2-phenylindole (DAPI) (1:5000; \#34007971; Dojindo Laboratories, Kumamoto, Japan). Membrane ruffling was visualized by staining of polymerized actin with phalloidin. Images were acquired by fluorescent microscopy (BZ-9000; Keyence, Osaka, Japan). Percentage of microglial ruffling was analyzed from 150 cells over three independent experiments. Line scan analysis was performed using ImageJ software $(\mathrm{NIH}$, Bethesda, MD, USA).

\section{Time-lapse imaging and migration analysis of cultured microglia}

Time-lapse imaging was performed as previously described [23]. Microglia were directly plated onto glass based dishes (\#3971-035; Iwaki, Tokyo, Japan). Images were collected at 2-min intervals using an imaging microscope system (LCV110; Olympus, Tokyo, Japan). Cells were maintained at $37^{\circ} \mathrm{C}$ in $5 \% \mathrm{CO}_{2}$ throughout the experiment. Time-lapse images were used for quantitative analysis of morphology, as well as migration velocity and distance. Morphological changes were analyzed from 30 cells pooled from three individual experiments. Speed and distance of microglial migration were determined by tracking microglial cell bodies using MetaMorph 7.5.6.0 software (Universal Imaging, Media, PA, USA).

\section{Measurement of intracellular CAMP accumulation}

Microglial cells were seeded at $3 \times 10^{4}$ cells/well in 96well plates and serum-starved for $4 \mathrm{~h}$. Microglia were stimulated with 6-OAU for $30 \mathrm{~min}$, subsequently treated with $50 \mu \mathrm{M}$ forskolin (Calbiochem, La Jolla, CA, USA) for $20 \mathrm{~min}$, and then extracted with $0.1 \mathrm{M}$ hydrochloric acid. Extracts were centrifuged at $1000 \times g$ for $10 \mathrm{~min}$, and supernatants were analyzed using the cAMP EIA kit (Cayman Chemical, Ann Arbor, MI, USA).

\section{Mutation verification}

mRNA was isolated from DBA/2 mice, and cDNA obtained by reverse transcription. One primer pair was used-forward: 5'-GCTCAGATGCCAACTTCTCC-3' and reverse: 5'-GACAACTGGCACCAAGACAA-3'. PCR was performed according to the manufacturer's instructions (LA Taq; \#RR002; Takara Bio, Otsu, Japan). PCR products were subjected to gel electrophoresis and bands collected for purification using the QIAquick gel extraction kit (\#28704; Qiagen, Valencia, CA, USA). Extracted DNA was sequenced using the Big Dye Terminator Cycle Sequencing Ready Reaction Kit (Applied Biosystems, Foster City, CA, USA). Sequencing was performed using an ABI PRISM 310 Genetic Analyzer (Applied Biosystems) and the primer, 5'AACAGCTCAGATGCCAACTTCTCCTGC-3'.

\section{Statistical analysis}

All data are shown as mean \pm standard error of the mean (SEM.). Statistical analyses were performed by one-way analysis of variance (ANOVA) with least significant difference (LSD), Dunnett's (E) or T3 (3) tests using SPSS 20. For single comparisons between two groups, 
unpaired Student's $t$ test was used. Differences were considered significant for values of $p<0.05$.

\section{Results \\ GPR84 does not alter pro-inflammatory responses of cul- tured microglia}

Previous studies have reported a pro-inflammatory role for GPR84 in macrophages $[17,18]$. Accordingly, we examined mRNA expression levels of pro-inflammatory cytokines including IL-1 $\beta$, IL-6, IL-12 subunit p35 and p40, CXCL1 (a murine homolog of IL-8), TNF- $\alpha$, and iNOS in LPS-treated primary cultured microglia after GPR84 agonist stimulation. Among surrogate agonists for GPR84, 6-OAU was used because of its potent activity [18]. Of all pro-inflammatory cytokines examined, mRNA expression was not up-regulated by 6-OAU treatment compared with vehicle. Rather, 6-OAU significantly suppressed mRNA expression of IL-6 (1 $\mu \mathrm{M}$, $p=0.019)$ and IL-12 p40 (0.1 $\mu \mathrm{M}, p=0.02)$ (Fig. 1a). Next, we examined additional inflammation-associated markers, specifically NF-kB. Nuclear translocation of NF- $\kappa B$ plays a critical role in pro-inflammatory gene expression [25, 26], therefore we examined subcellular localization of the NF- $\mathrm{BB}$ RelA subunit (p65) after 6OAU treatment. Under normal culture conditions, p65 was spread throughout both the cytoplasm and nucleus of microglia, while LPS treatment prominently induced p65 translocation into the nucleus. In agreement with mRNA expression, 6-OAU as well as other GPR84 ligands, namely capric acid and embelin, did not cause nuclear translocation of p65 in microglia (Fig. 1b). Line scanning analysis determined the fluorescence intensity of intracellular p65 localization. p65 remained in the cytoplasm even after stimulation with GPR84, in contrast to nuclear localization in LPS-treated microglia (Fig. 1c). Taken together, GPR84-mediated signaling does not induce a pro-inflammatory phenotype in microglia in vitro.

\section{GPR84 agonists rapidly induce microglial ruffling}

Next, we examined morphological changes in microglia (Fig. 2). 6-OAU induced apparent lamellipodia formation in cultured microglia (Fig. 2a, b). Microglia exhibited rounded cell bodies, with the cytoplasmic membrane showing sheet-like membrane protrusions that were heavily stained with phalloidin. Microglial ruffling was also observed using the endogenous ligand, capric acid, and a natural ligand, embelin. The percentage of microglial rufflings was calculated by staining actinpolymerized lamellipodia with phalloidin [27]. Two minutes after agonist application, the highest percentage of ruffling was observed with 6-OAU (6-OAU at $1 \mu \mathrm{M}$, $86 \%$; capric acid at $1 \mu \mathrm{M}, 23 \%$; embelin at $1 \mu \mathrm{M}, 26.4 \%$ ) (Fig. 2c-e). Efficiency of the three agonists on microglial ruffling correlates with cAMP suppression activity shown in a previous study [18]. Thus, we primarily used 6-OAU for further research.

\section{Microglial motility is induced by GPR84 agonists}

Membrane ruffling is associated with microglial motility [28], therefore we examined motility of 6-OAU-treated microglia using time-lapse imaging (Fig. 3). 6-OAU induced ruffling, as shown in Fig. 2. 6-OAU treatment also increased microglial motility over a relatively short period, which was demonstrated by re-plotting microglial migration trajectories (Fig. 3b, c). Measurement of distance and velocity clearly indicated that 6-OAU induced higher motility (Fig. 4a, b). A similar response was observed in capric acid- (Fig. 4c, d) and embelin(Fig. 4e, f) treated microglia, indicating that GPR84mediated signals enhance microglial motility.

\section{6-OAU-induced microglial motility is mediated by a PTX- sensitive Gi/o pathway}

GPR84 activation by its ligands is coupled to a PTXsensitive Gi/o pathway [18]. Hence, we examined the involvement of the Gi/o pathway. PTX completely suppressed 6-OAU-induced microglial motility, such as distance and velocity (Fig. $5 \mathrm{a}-\mathrm{C}$ ). We also found that membrane ruffling of cultured microglia exposed to 6OAU was completely blocked (Fig. 5d). In support of Gi/ o-coupling of GPR84, we confirmed that 6-OAU dosedependently inhibited forskolin-stimulated cAMP production in cultured microglia (Fig. 5e). Altogether, these results suggest that GPR84 activation by 6-OAU couples primarily to a PTX-sensitive Gi/o pathway.

\section{GPR84-deficient microglia fail to respond to 6-OAU}

To further confirm that motility changes induced by 6OAU are GPR84 dependent, we used the DBA/2 mouse strain, which has a 2-bp frame-shift deletion in the second exon of the GPR84 gene. The deletion results in a premature stop codon and predicted truncated protein [11]. We sequenced exon 2 of GPR84 using DBA/2 mice cDNA to confirm the 2-bp frame-shift deletion (Fig. 6a). 6-OAU-induced membrane ruffling did not occur in microglia from DBA/2 mice (Fig. 6b, c). As expected, 6OAU-induced motility was also blocked, with migration distance and velocity unchanged after 6-OAU treatment in DBA/2 microglia (Fig. 6d-g). These findings indicate that GPR84 is involved in microglial ruffling and motility induced by 6-OAU.

\section{Discussion}

In the present study, we show that GPR84 mediated signaling induces microglial ruffling and motility via the Gi/o pathway (Figs. 2, 3, 4, 5, and 6), but does not alter pro-inflammatory responses in vitro. These responses 


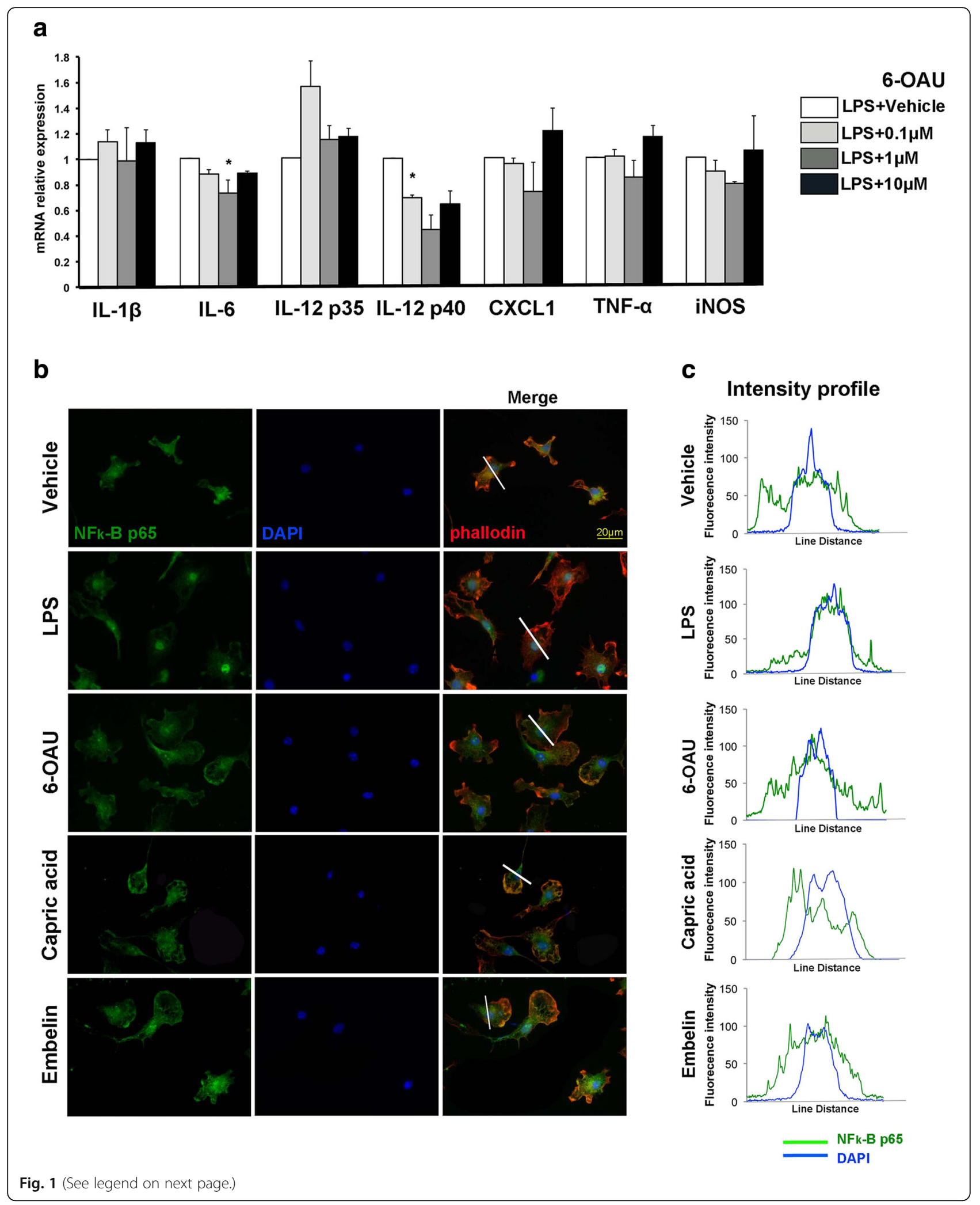


(See figure on previous page.)

Fig. 1 GPR84 does not modulate pro-inflammatory responses in cultured microglia. a Primary cultured microglia were treated with bacterial lipopolysaccharide (LPS) and either vehicle or 6-OAU for $16 \mathrm{~h}$. mRNA for interleukin (IL)-1 $\beta$, IL-6, IL-12 p35, IL-12 p40, chemokine (C-X-C motif) ligand 1 (CXCL1), tumor necrosis factor (TNF)- $\alpha$, and inducible nitric oxide synthase (iNOS) was quantified by quantitative real-time RT-PCR. Results were normalized to LPS-treated samples. Values represent mean \pm SEM from three independent experiments. ${ }^{*} p<0.05$. $\mathbf{b}$ Microglia were treated with vehicle, $100 \mathrm{ng} / \mathrm{mL}$ LPS, $1 \mu \mathrm{M}$ 6-OAU, $1 \mu \mathrm{M}$ capric acid, and $1 \mu \mathrm{M}$ embelin for $16 \mathrm{~h}$. Cells were stained with anti-nuclear factor kappa B (NF-KB) p65 antibody (green), Alexa Fluor 594-labeled phalloidin (red), and DAPI (blue). c Line scan graphs of representative cells show the fluorescent intensity of p65 (green) and DAPI (blue) along white lines. Scale bar, $20 \mu \mathrm{m}$

may be characteristic of GPR84 signaling in microglia, and distinct from macrophages.

GPR84 is a seven-transmembrane GPCR, initially identified as an orphan receptor [9]. Later, the $\left[{ }^{35} \mathrm{~S}\right] \mathrm{GTP} \gamma \mathrm{S}$ binding assay, which can detect Goi pathways, demonstrated that medium-chain fatty acids, especially capric acid (a 10 carbon chain fatty acid), have the most potent activity for GPR84 in vitro [17]. Expression of GPR84 is mainly seen in myeloid cells (e.g., monocytes, macrophages, microglia, and neutrophils) and is up-regulated by immune stimuli $[7,29,30]$. In the central nervous system (CNS), several transcriptome studies have reported GPR84 as one of the signature microglial genes [31, 32]. In addition, peripheral nerve injury induced GPR84 expression in microglia [3,13], leading us to propose that GPR84 is important for microglial regulation, although its precise function remains to be determined.
Potent surrogate agonists are a useful tool for characterizing functionally unknown or less known GPCRs. Suzuki et al. [18] screened an in-house chemical library with the $\left[{ }^{35} \mathrm{~S}\right] \mathrm{GTP} \gamma \mathrm{S}$ binding assay and identified a highly effective agonist compound, 6-OAU, which activated human GPR84 in the presence of Gqi5 chimeric G proteins. Furthermore, EGFP-labeled human GPR84 internalization was observed in a 6-OAU-dependent manner. Altogether, these findings suggest that 6-OAU activates GPR84 [18]. GPR84 is proposed to be a pro-inflammatory receptor that induces pro-inflammatory cytokine expression [13, 17, 18]. GPR84 is expressed selectively in immune cells [7, 17], and Suzuki et al. have shown that GPR84 activation amplifies LPS-stimulated IL-8 production in human polymorphonuclear leukocytes and TNF- $\alpha$ production in macrophages [18]. Concomitantly in a peripheral nerve injury-induced neuropathic pain model, GPR84 deficiency

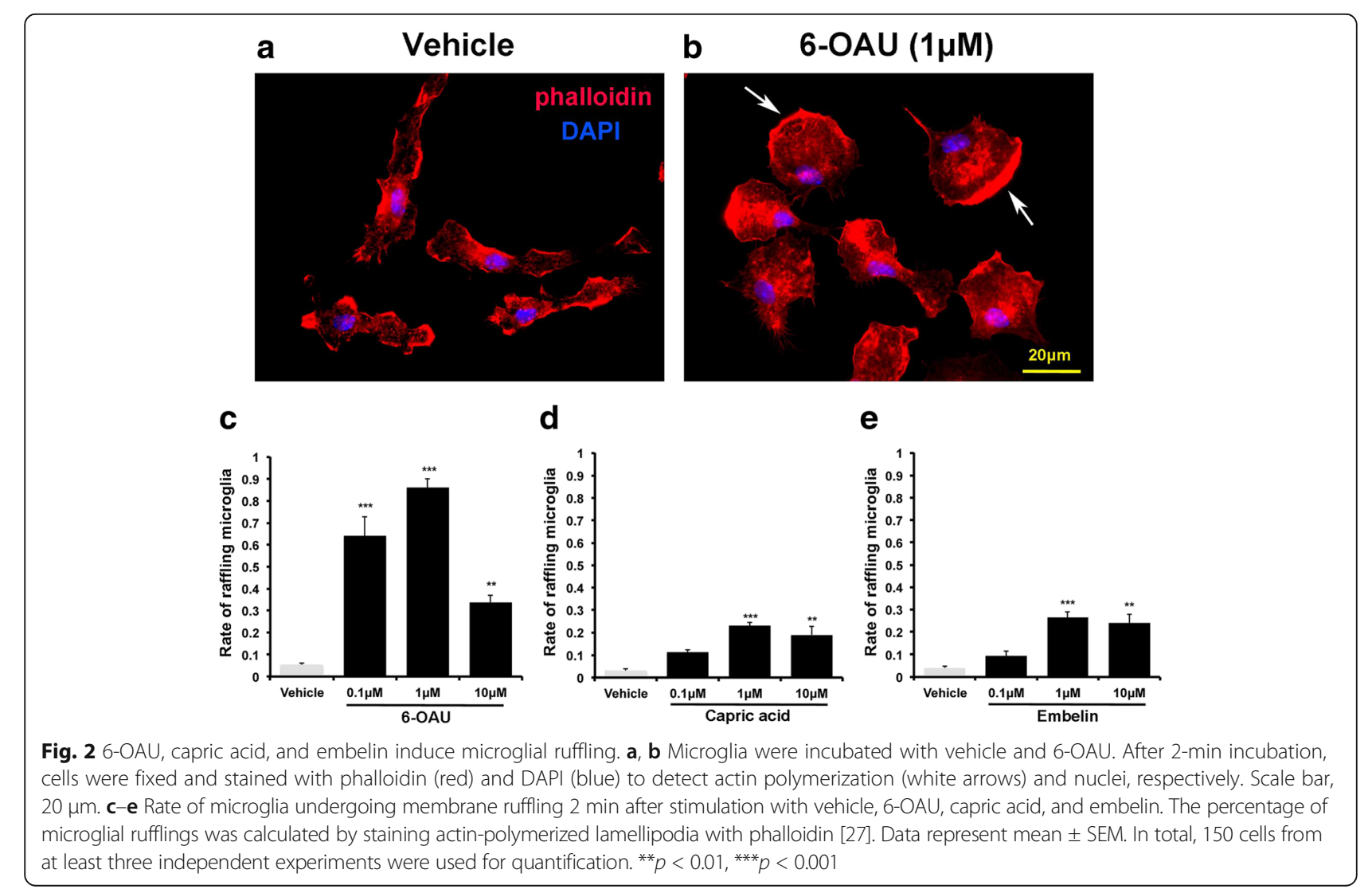




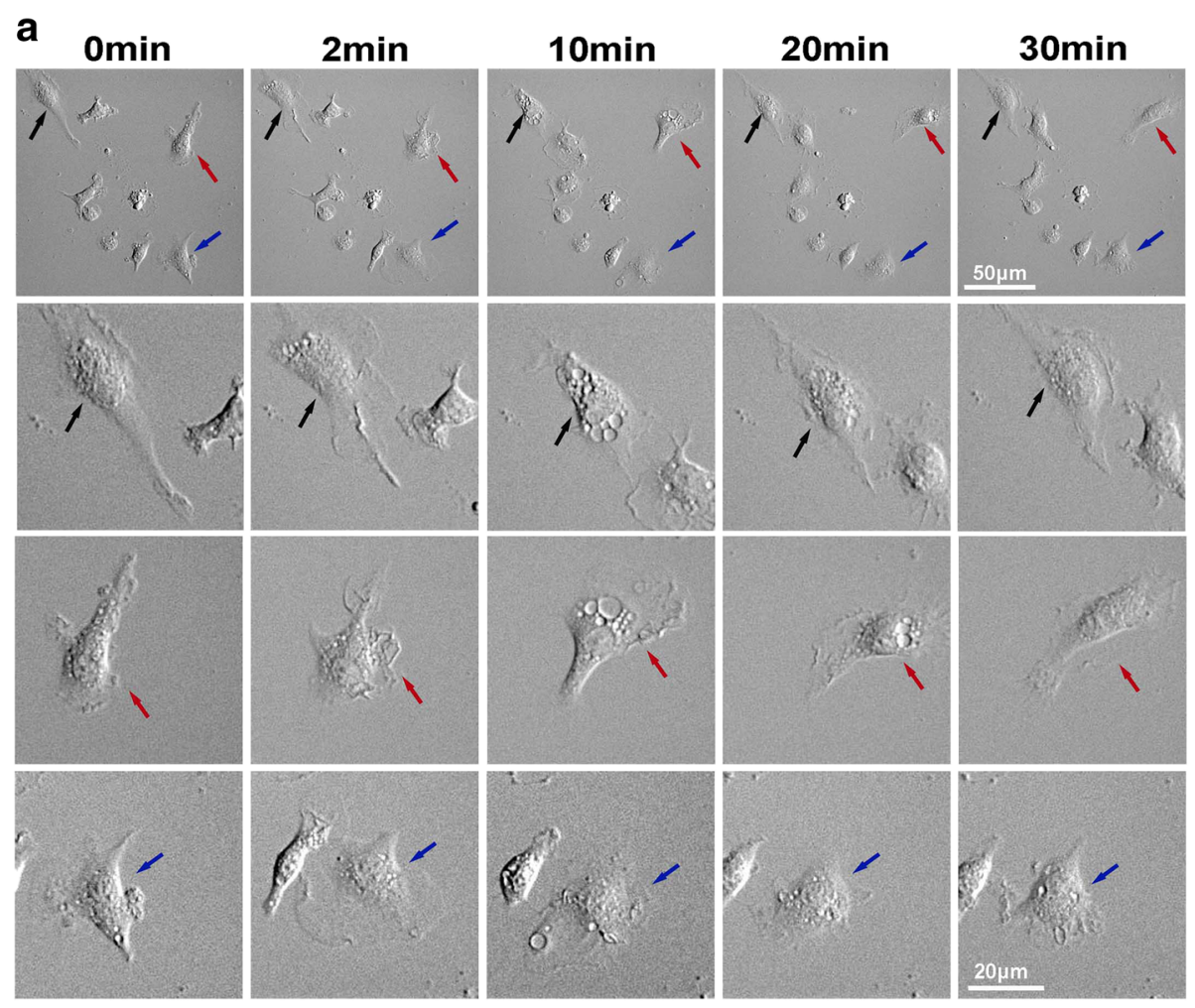

b

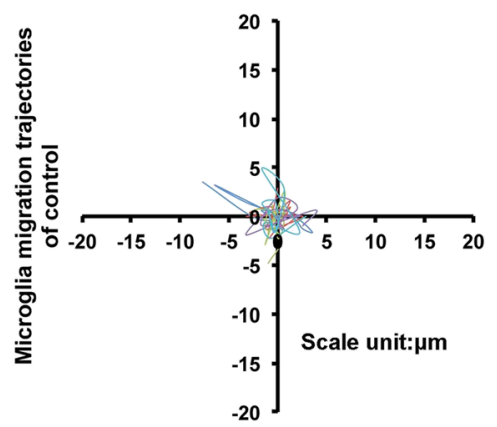

C

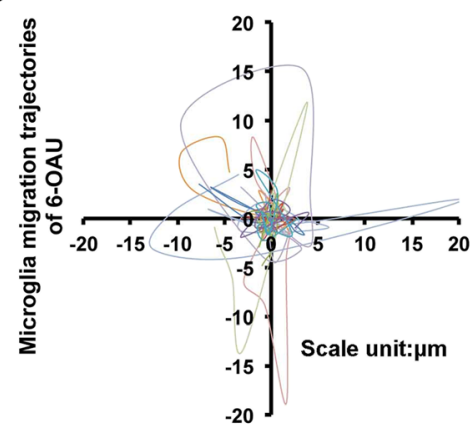

Fig. 3 Time-lapse analysis of 6-OAU-treated microglia. a Microglia were exposed to $1 \mu \mathrm{M}$ 6-OAU, and live imaging was performed for 30 min. The second, third, and fourth rows show higher magnifications of cells indicated by arrows in the top row. Scale bar, $50 \mu \mathrm{m}$ (top row) and $20 \mu \mathrm{m}$ (other rows). b, c Overlay of individual microglial trajectories after control or 6-OAU treatment for $30 \mathrm{~min}$ ( $n=5$ each). The starting point of each cell was aligned to the same position $(x=0, y=0)$

induced arginase 1 expression in macrophages that infiltrated peripheral nerve and reduced neuropathic pain behavior [13]. Collectively these experiments suggest that GPR84 mediates pro-inflammatory signaling, at least in macrophages. However, in microglia, mRNA expression of pro-inflammatory cytokines was not induced by the agonists, 6-OAU, capric acid, and embelin (Fig. 1a). In addition, these agonists did not cause nuclear translocation of NF- $\mathrm{BB}$, which elicits inflammatory microglial responses (Fig. 1b) [26, 33], suggesting that GPR84 is not a pro-inflammatory receptor in microglia. Instead, here we demonstrate GPR84 as an activator of motility in microglia (Figs. 2, 3, 4, 5, and 6). Because Suzuki et al. showed that 6-OAU stimulates chemotaxis of macrophages both in vitro and in vivo [18], induction of motility by GPR84 is shared between macrophages and microglia. GPR84 uses a PTX-sensitive Gi/o downstream signaling pathway to induce motility of both macrophages [18] and microglia [8] (Fig. 5). Although downstream receptor signaling appears to be the same between microglia and macrophages, microglial inflammatory responses failed to respond to agonist stimuli (Fig. 1). We cannot explain this, but 

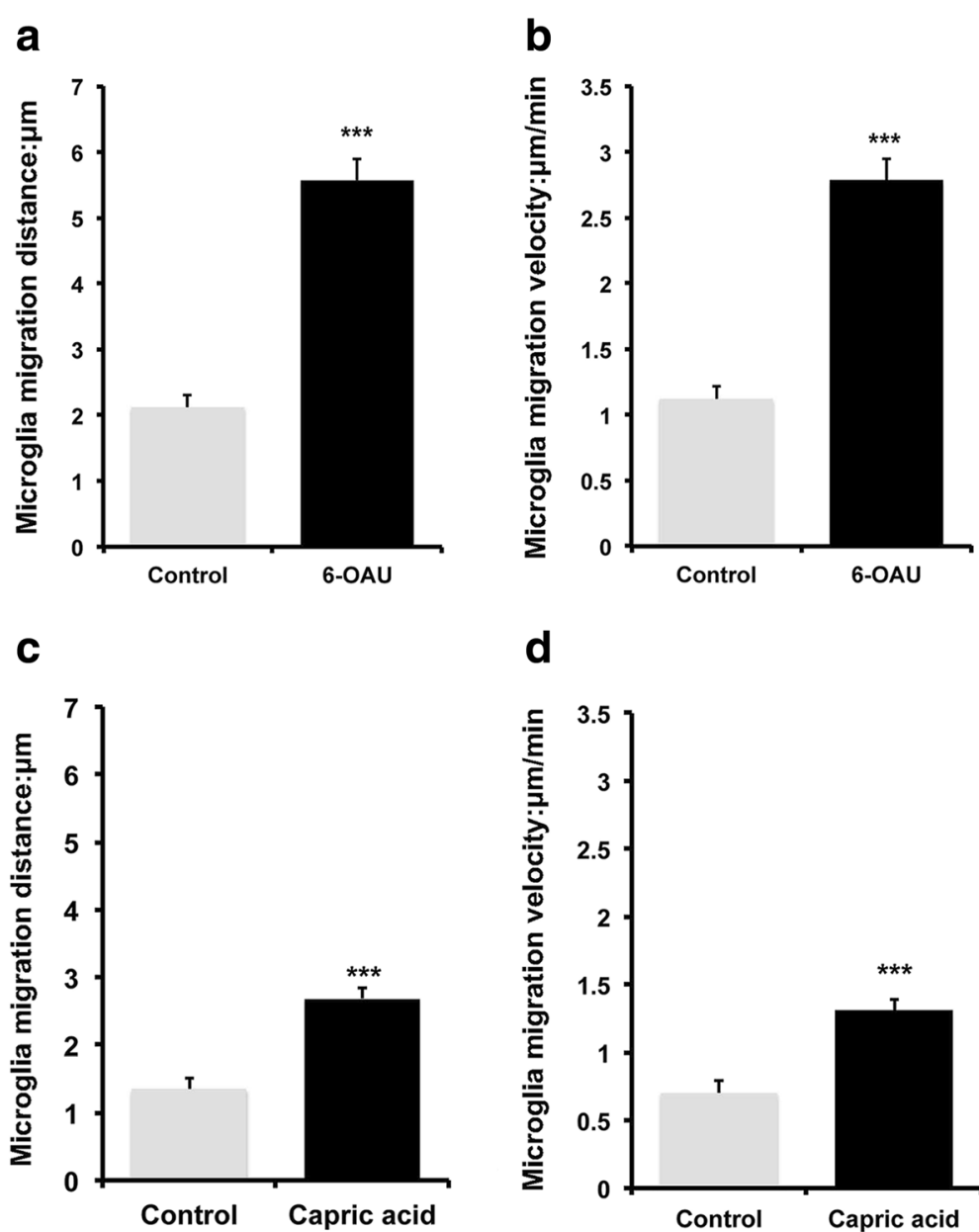

d
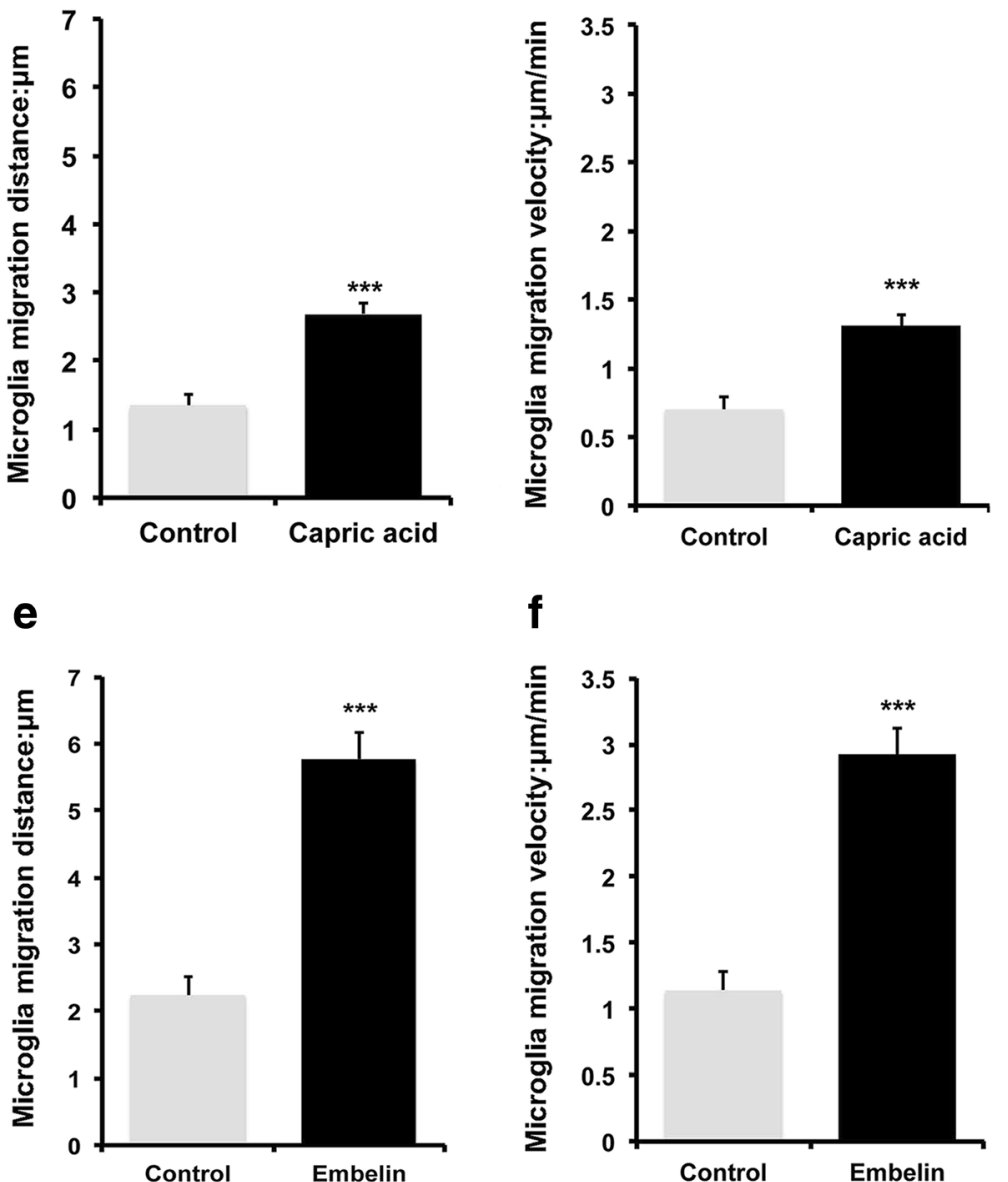

Fig. 4 Quantification of cell migration distance and velocity. Cells were tracked over 30-min periods and MetaMorph-based numerical values calculated. Microglia were exposed to $1 \mu \mathrm{M}$ 6-OAU $(\mathbf{a}, \mathbf{b})$, capric acid $(\mathbf{c}, \mathbf{d})$, and embelin $(\mathbf{e}$, $\mathbf{f})$, with migration distance $(\mathbf{a}, \mathbf{c}, \mathbf{e})$ and velocity $(\mathbf{b}, \mathbf{d}, \mathbf{f})$ calculated. Data represent mean \pm SEM of $15(\mathbf{a}, \mathbf{b}), 13(\mathbf{c}, \mathbf{d})$, and $30(\mathbf{e}, \mathbf{f})$ cells per group. ${ }^{* * *} p<0.001$

additional unknown signaling pathways may exist downstream of the receptor.

Several fatty acids regulate microglial morphology and activity [19-21]. Given that medium-chain fatty acids are endogenous ligands for GPR84 [17, 18], fatty acids released under inflammatory, traumatic, and degenerative events within the CNS [34-36] may trigger activation of microglial GPR84. In this context, microglial GPR84 


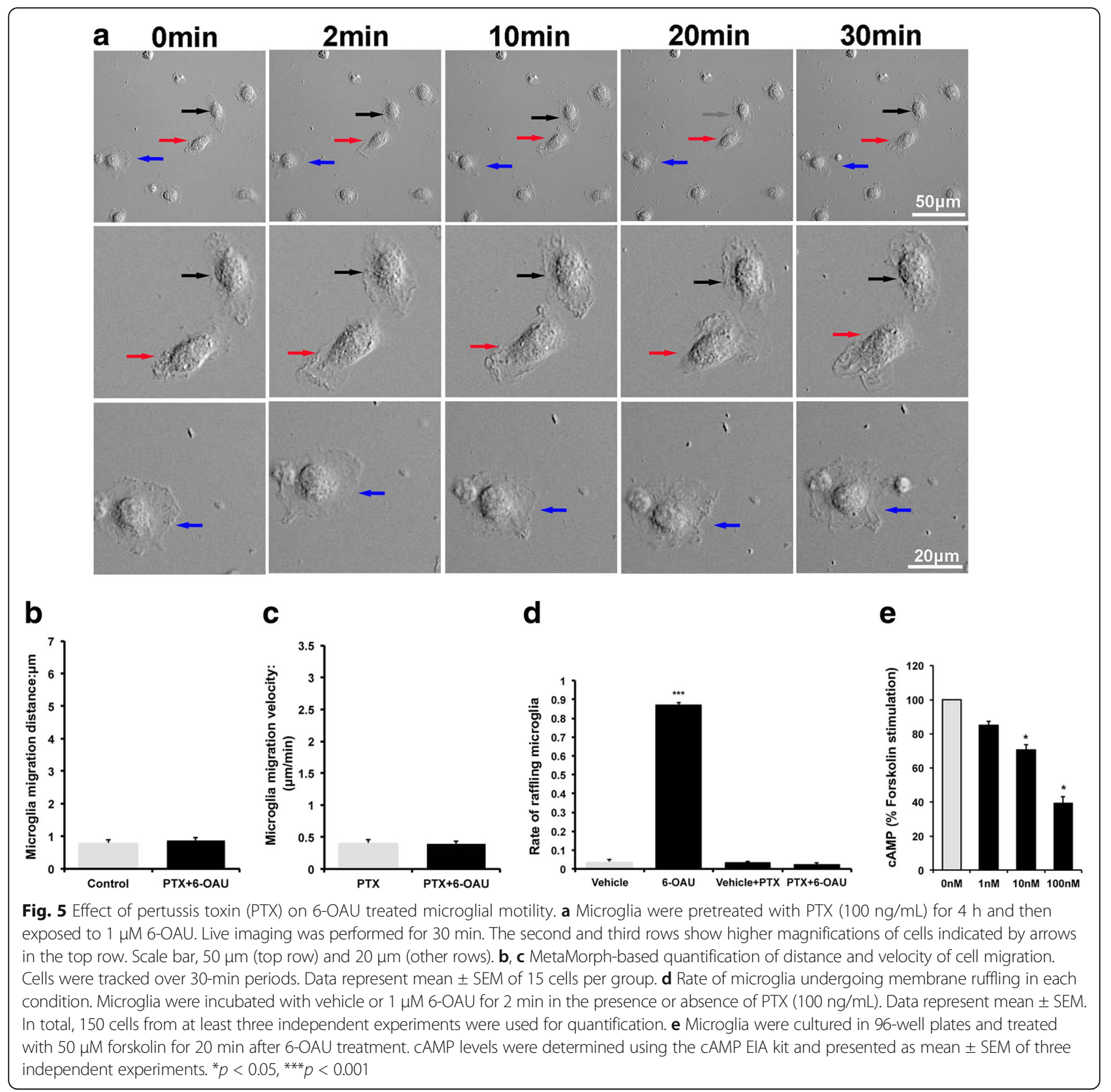

appears to be involved in activation of microglial motility in response to CNS insults [8]. This is suggested by the fact that peripheral nerve injury promotes GPR84 expression in microglia and induces microglial migration towards injured neurons [3].

This study demonstrates the functional significance of GPR84 agonists in microglia in vitro. Our findings show that GPR84 agonist-mediated signaling affects morphology and motility of microglia, suggesting that microglial GPR84 could be a therapeutic target in microgliaassociated diseases such as multiple sclerosis and
Alzheimer's disease [8]. Further development of both agonists and antagonists will provide increased understanding of GPR84 function as well as therapeutic tools for neuronal inflammatory, traumatic, and degenerative diseases.

\section{Conclusions}

The present study demonstrates that GPR84 does not modulate pro-inflammatory responses in primary cultured microglia. However, endogenous and natural ligands and a surrogate agonist (capric acid, embelin, 


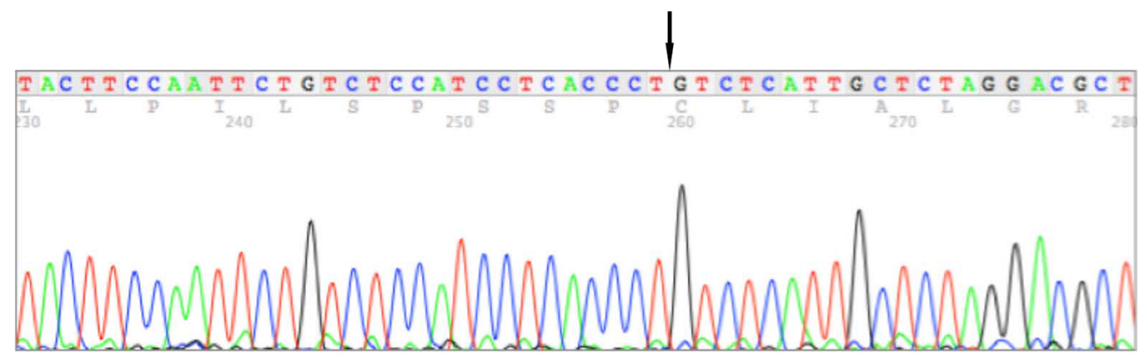

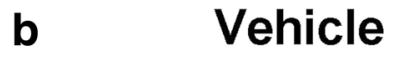

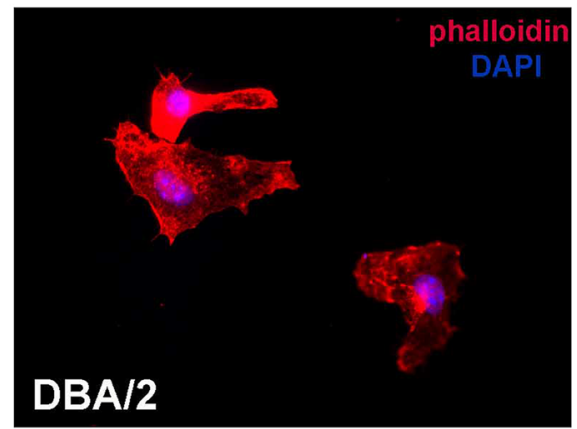

d

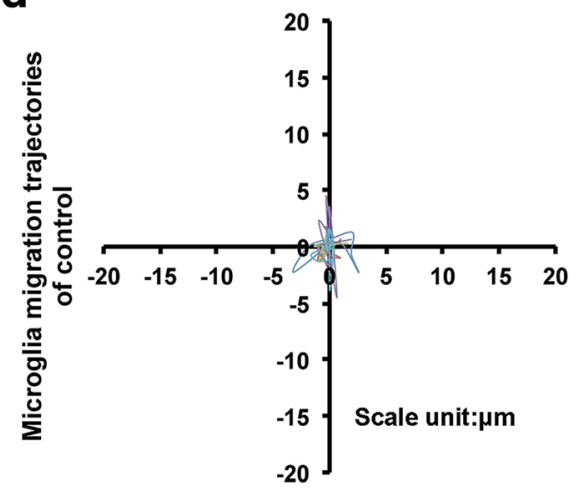

f

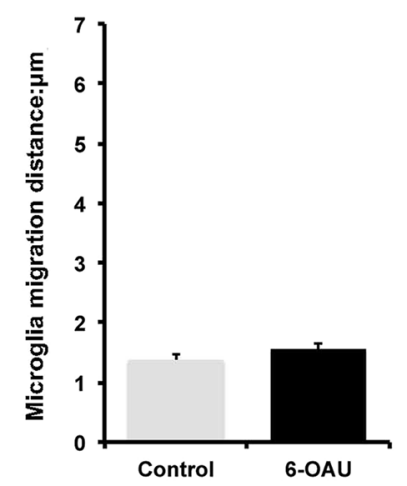

\section{g}

c 6-OAU

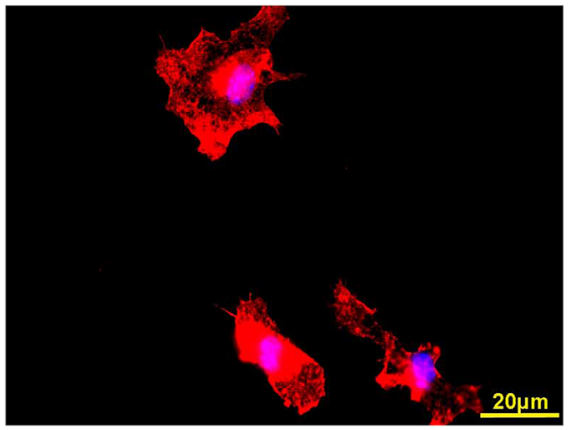

e

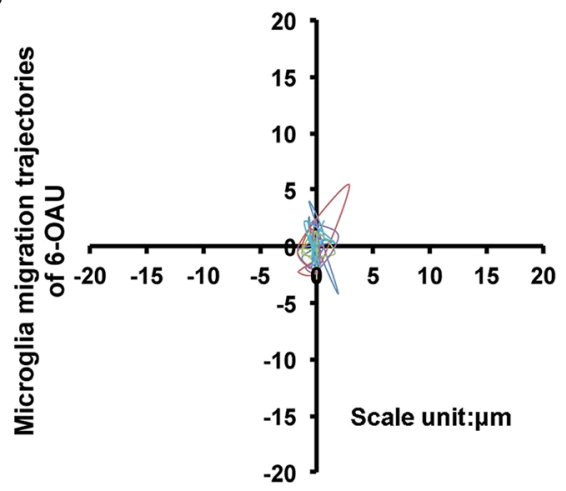

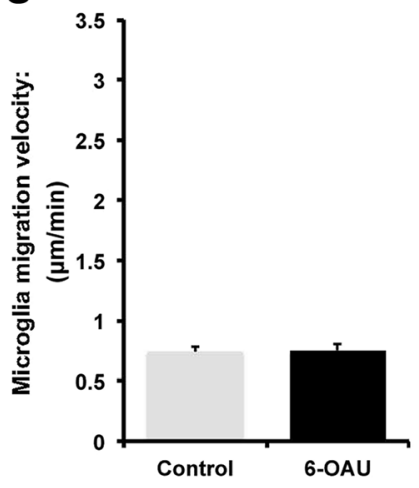

Fig. 6 (See legend on next page.) 
(See figure on previous page.)

Fig. 6 Motility analysis of 6-OAU-treated microglia from DBA/2 mice. a DNA sequencing shows a frame-shift deletion in exon 2 of GPR84 from DBA/2 mice. b, c Microglia from DBA/2 mice were exposed to vehicle and $1 \mu \mathrm{M}$ 6-OAU. After 2-min incubation, cells were fixed and stained with phalloidin (red) and DAPI (blue) to detect actin polymerization and nuclei, respectively. Scale bar, $20 \mu \mathrm{m}$. d, e Overlay of individual microglial trajectories after vehicle or 6-OAU treatment for $30 \mathrm{~min}(n=5$ each). $\mathbf{f}, \mathbf{g}$ MetaMorph-based quantification of distance and velocity of cell migration. Cells were tracked over 30-min periods. Data represent mean \pm SEM of 10 cells per group

and 6-OAU, respectively) for GPR84 rapidly induce microglial ruffling and motility. 6-OAU induced activation of microglia was suppressed by PTX, which inhibits the Gi/o pathway located downstream of GPR84. Concomitantly, 6-OAU failed to induce membrane ruffling and motility in primary cultured microglia from DBA/2 mice lacking functional GPR84. Since GPR84 is known as a receptor for medium-chain fatty acids such as capric acid, fatty acids released from damaged brain cells may act on microglial GPR84 to increase cellular motility.

\section{Acknowledgements}

We appreciate Ms. Ayako Asano for her excellent secretary service, and all members of the Kiyama lab at Nagoya University for their help and kindness. We thank Ono Pharmaceutical Co., Ltd., for providing 6-OAU, embelin, and capric acid.

\section{Funding}

This work was supported by the Japan Society for the Promotion of Science (JSPS) RONPAKU Program (R1 1402), a Grant-in-aid for Scientific Research from JSPS (16K15170), Japan-China Medical Association, and Chongqing Science and Technology Committee (CSTC2016JCYJA0030 and 2016cstc-jbky-01702).

\section{Availability of data and materials}

Please contact the author for data requests.

\section{Authors' contributions}

LW and KT designed the study, performed most of the experiments and statistical analysis, and drafted the manuscript. HY participated in the study design. HKo participated in the study design and the experiments. HKi conceived the project, supervised the experiments, and made significant contributions to editing and revising

the paper. All authors read and approved the final manuscript.

\section{Ethics approval and consent to participate}

Not applicable

Consent for publication

Not applicable

\section{Competing interests}

The authors declare that they have no competing interests.

\section{Publisher's Note}

Springer Nature remains neutral with regard to jurisdictional claims in published maps and institutional affiliations.

\section{Author details}

'Department of Functional Anatomy and Neuroscience, Nagoya University, Graduate School of Medicine, Nagoya, 65 Tsurumai-cho, Showa-ku, Nagoya, Aichi 466-8550, Japan. ${ }^{2}$ National Key Laboratory of Birth Defects and Reproductive Health, Chongqing Institute of Population and Family Planning, Chongqing 400020, China. ${ }^{3}$ College of Basic Medical Science, Chongqing Medical University, Chongqing 400016, China.
Received: 5 June 2017 Accepted: 25 September 2017

Published online: 03 October 2017

\section{References}

1. Fields RD, Burnstock G. Purinergic signalling in neuron-glia interactions. Nat Rev Neurosci. 2006:7:423-36.

2. Old EA, Malcangio M. Chemokine mediated neuron-glia communication and aberrant signalling in neuropathic pain states. Curr Opin Pharmacol. 2012;12:67-73.

3. Gamo K, Kiryu-Seo S, Konishi H, Aoki S, Matsushima K, Wada K, Kiyama H. Gprotein-coupled receptor screen reveals a role for chemokine receptor CCR5 in suppressing microglial neurotoxicity. J Neurosci. 2008;28:1 1980-8.

4. Maeda M, Tsuda M, Tozaki-Saitoh H, Inoue K, Kiyama H. Nerve injuryactivated microglia engulf myelinated axons in a P2Y12 signalingdependent manner in the dorsal horn. Glia. 2010:58:1838-46.

5. Tsuda M, Shigemoto-Mogami Y, Koizumi S, Mizokoshi A, Kohsaka S, Salter MW, Inoue K. P2X(4) receptors induced in spinal microglia gate tactile allodynia after nerve injury. Nature. 2003;424:778-83.

6. Cardona AE, Pioro EP, Sasse ME, Kostenko V, Cardona SM, Dijkstra IM, Huang DR, Kidd G, Dombrowski S, Dutta R, et al. Control of microglial neurotoxicity by the fractalkine receptor. Nat Neurosci. 2006;9:917-24.

7. Bouchard C, Page J, Bedard A, Tremblay P, Vallieres L. G protein-coupled receptor 84 , a microglia-associated protein expressed in neuroinflammatory conditions. Glia. 2007:55:790-800.

8. Audoy-Remus J, Bozoyan L, Dumas A, Filali M, Cynthia L, Lacroix S, Rivest S, Tremblay ME, Vallieres L. GPR84 deficiency reduces microgliosis, but accelerates dendritic degeneration and cognitive decline in a mouse model of Alzheimer's disease. Brain Behav Immun. 2015:46:112-20.

9. Wittenberger T, Schaller HC, Hellebrand S. An expressed sequence tag (EST) data mining strategy succeeding in the discovery of new G-protein coupled receptors. J Mol Biol. 2001;307:799-813.

10. Yousefi S, Cooper PR, Potter SL, Mueck B, Jarai G. Cloning and expression analysis of a novel G-protein-coupled receptor selectively expressed on granulocytes. J Leukoc Biol. 2001;69:1045-52.

11. Perez CJ, Dumas A, Vallieres L, Guenet JL, Benavides F. Several classical mouse inbred strains, including DBA/2, NOD/Lt, FVB/N, and SJL/J, carry a putative loss-of-function allele of Gpr84. J Hered. 2013:104:565-71.

12. Venkataraman C, Kuo F. The G-protein coupled receptor, GPR84 regulates IL-4 production by T lymphocytes in response to CD3 crosslinking. Immunol Lett. 2005;101:144-53.

13. Nicol LSC, Dawes JM, La Russa F, Didangelos A, Clark AK, Gentry C, Grist J, Davies JB, Malcangio M, McMahon SB. The role of G-protein receptor 84 in experimental neuropathic pain. J Neurosci. 2015;35:8959-69.

14. Tashima R, Mikuriya S, Tomiyama D, Shiratori-Hayashi M, Yamashita T, Kohro $\mathrm{Y}$, Tozaki-Saitoh H, Inoue K, Tsuda M. Bone marrow-derived cells in the population of spinal microglia after peripheral nerve injury. Sci Rep. 2016;6:8

15. Kobayashi M, Konishi H, Sayo A, Takai T, Kiyama H. TREM2/DAP12 signal elicits proinflammatory response in microglia and exacerbates neuropathic pain. J Neurosci. 2016;36:11138-50.

16. Ajami B, Bennett JL, Krieger C, Tetzlaff W, Rossi FMV. Local self-renewal can sustain CNS microglia maintenance and function throughout adult life. Nat Neurosci. 2007;10:1538-43.

17. Wang JH, Wu XS, Simonavicius $\mathrm{N}$, Tian H, Ling L. Medium-chain fatty acids as ligands for orphan G protein-coupled receptor GPR84. J Biol Chem. 2006: 281:34457-64.

18. Suzuki M, Takaishi S, Nagasaki M, Onozawa Y, lino I, Maeda H, Komai T, Oda T. Medium-chain fatty acid-sensing receptor, GPR84, is a proinflammatory receptor. J Biol Chem. 2013;288:10684-91.

19. Erny D, de Angelis ALH, Jaitin D, Wieghofer P, Staszewski O, David E, KerenShaul H, Mahlakoiv T, Jakobshagen K, Buch T, et al. Host microbiota constantly control maturation and function of microglia in the CNS Nat Neurosci. 2015;18:965. 
20. Sampson TR, Debelius JW, Thron T, Janssen S, Shastri GG, Ilhan ZE, Challis C, Schretter CE, Rocha S, Gradinaru V, et al. Gut microbiota regulate motor deficits and neuroinflammation in a model of Parkinson's disease. Cell. 2016; 167:1469.

21. Guida F, Luongo L, Boccella S, Giordano ME, Romano R, Bellini G, Manzo I, Furiano A, Rizzo A, Imperatore R, et al. Palmitoylethanolamide induces microglia changes associated with increased migration and phagocytic activity: involvement of the CB2 receptor. Sci Rep. 2017;7:11.

22. Konishi H, Namikawa K, Kiyama H. Annexin III implicated in the microglial response to motor nerve injury. Glia. 2006;53:723-32.

23. Tokizane K, Konishi H, Makide K, Kawana H, Nakamuta S, Kaibuchi K, Ohwada T, Aoki J, Kiyama H. Phospholipid localization implies microglial morphology and function via Cdc42 in vitro. Glia. 2017;65:740-55.

24. Tokizane K, Konishi H, Yasui M, Ogawa T, Sasaki K, Minamino N, Kiyama H. Continuous stress promotes expression of VGF in melanotroph via suppression of dopamine. Mol Cell Endocrinol. 2013;372:49-56.

25. May MJ, Ghosh S. Signal transduction through NF-kappa B. Immunol Today. 1998;19:80-8.

26. Bonizzi G, Karin M. The two NF-kappa B activation pathways and their role in innate and adaptive immunity. Trends Immunol. 2004;25:280-8.

27. Haynes SE, Hollopeter G, Yang G, Kurpius D, Dailey ME, Gan WB, Julius D. The P2Y(12) receptor regulates microglial activation by extracellular nucleotides. Nat Neurosci. 2006;9:1512-9.

28. Honda K, Shimohama S, Sawada H, Kihara T, Nakamizo T, Shibasaki H, Akaike A. Nongenomic antiapoptotic signal transduction by estrogen in cultured cortical neurons. J Neurosci Res. 2001:64:466-75.

29. Ichimura A, Hirasawa A, Hara T, Tsujimoto G. Free fatty acid receptors act as nutrient sensors to regulate energy homeostasis. Prostaglandins Other Lipid Mediat. 2009;89:82-8.

30. Oh DY, Lagakos WS. The role of G-protein-coupled receptors in mediating the effect of fatty acids on inflammation and insulin sensitivity. Curr Opin Clin Nutr Metab Care. 2011;14:322-7.

31. Gautier EL, Shay T, Miller J, Greter M, Jakubzick C, Ivanov S, Helft J, Chow A, Elpek KG, Gordonov S, et al. Gene-expression profiles and transcriptional regulatory pathways that underlie the identity and diversity of mouse tissue macrophages. Nat Immunol. 2012;13:1118-28.

32. Hickman SE, Kingery ND, Ohsumi TK, Borowsky ML, Wang LC, Means TK, El Khoury J. The microglial sensome revealed by direct RNA sequencing. Nat Neurosci. 2013;16:1896-905.

33. Frakes AE, Ferraiuolo L, Haidet-Phillips AM, Schmelzer L, Braun L, Miranda CJ, Ladner KJ, Bevan AK, Foust KD, Godbout JP, et al. Microglia induce motor neuron death via the classical NF-kappa B pathway in amyotrophic lateral sclerosis. Neuron. 2014;81:1009-23.

34. Clemens JA, Stephenson DT, Smalstig EB, Roberts EF, Johnstone EM, Sharp JD, Little SP, Kramer RM. Reactive glia express cytosolic phospholipase A2 after transient global forebrain ischemia in the rat. Stroke. 1996;27:527-35.

35. Anthonymuthu TS, Kenny EM, Bayir H. Therapies targeting lipid peroxidation in traumatic brain injury. Brain Res. 2016;1640:57-76.

36. Shohami E, Shapira Y, Yadid G, Reisfeld N, Yedgar S. Brain phospholipase A2 is activated after experimental closed head injury in the rat. J Neurochem. 1989:53:1541-6.

\section{Submit your next manuscript to BioMed Central and we will help you at every step:}

- We accept pre-submission inquiries

- Our selector tool helps you to find the most relevant journal

- We provide round the clock customer support

- Convenient online submission

- Thorough peer review

- Inclusion in PubMed and all major indexing services

- Maximum visibility for your research

Submit your manuscript at www.biomedcentral.com/submit

) Biomed Central 